\title{
WEAK TOPOLOGY AND NONLINEAR INTEGRAL EQUATIONS
}

BY

E. H. ROTHE

To the memory of A. Hammerstein

1. Introduction. A. Hammerstein was the first to develop a systematic theory of nonlinear integral equations in the large $\left({ }^{1}\right)$. He proved existence and uniqueness theorems for the equation $\left({ }^{2}\right)$

$$
y(s)+\int_{D} K(s, t) f(t, y(t)) d t=0
$$

in the case of a symmetric and positive definite kernel $K(s, t)$. M. Golomb $\left.{ }^{8}\right)$ discarded the assumptions of symmetry and definiteness by somewhat strengthening Hammerstein's hypotheses in other respects. He also treated systems of equations.

In the present paper an attempt is made to develop the theory of existence $\left(^{4}\right)$ of solutions of nonlinear integral equations from the point of view of the "weak" topology in a suitable function space. More specifically the results will be based on the following theorem.

THEOREM 1.1. Let $E$ be a reflexive Banach space and $V$ the solid sphere $\|x\| \leqq R$ where $\|x\|$ denotes the norm of the element $x$ of $E$, and $R$ is a positive number. Let $I(x)$ be a scalar (that is, a real-valued function) defined in $V$ and having a completely continuous Fréchet differential $\left(^{5}\right) D(x, h)$ in every point $x$ of $V$. Then $I(x)$ reaches an absolute maximum and an absolute minimum in $V$. Moreover the scalar

$$
i(x)=\|x\|^{2} / 2+I(x)
$$

reaches an absolute minimum in $V$.

This theorem was proved in a former paper [9, Theorems 4.1 and 4.2] by combining a well known theorem by Alaoglu [1, Theorem 1.3] with the

Presented to the Society, April 17, 1948; received by the editors February 17, 1948.

(1) [6]. The ramification theory of E. Schmidt [11] deals with problems in the small. (Numbers in brackets refer to the bibliography at the end of the paper.)

$\left.{ }^{2}\right) D$ is a domain in the Euclidean $r$-space, and $s, t$ denote points in $D$.

(3) [3], [4]. Golomb was also the first to derive existence theorems for nonlinear integral equations by the use of extrema of certain functionals in abstract spaces $[3, \S \delta 6,7]$, a method systematically used in the present paper.

${ }^{(4)}$ Questions of uniqueness are not treated in this paper.

(5) For the definition of complete continuity of $D(x, h)$ see [9, Definition 3.1]. 
fact [9, Theorem 3.2] that the assumptions of Theorem 1.1 imply the "weak" continuity of $I(x)$.

In the present treatment two scalars $i(x), i^{*}(x)$ of the form (1.2) will play a central role (Definition 3.1). They will be referred to as "Hammerstein scalars" since they are essentially an extension of the double integral

$$
\begin{gathered}
\frac{1}{2} \int_{D} \int_{D} K(s, t) x(s) x(t) d s d t+\int_{D} F\left(t, \int_{D} K(t, s) x(s) d s\right) d t \\
F(t, y)=\int_{0}^{y} f(t, u) d u
\end{gathered}
$$

with which Hammerstein dealt repeatedly $\left(^{6}\right)$. He proved (under certain conditions) the existence of a $y(t)$ minimizing (1.3) in case of a symmetric and positive definite kernel $K(s, t)$. Without any assumptions concerning definiteness or symmetry it will be shown in the present paper that the scalars $I(x)$, $I^{*}(x)$ (Definition 3.1) satisfy the hypotheses of Theorem 1.1 (see Theorem 3.2) which implies immediately the existence of a maximum and minimum for $I(x), I^{*}(x)$ and of a minimum for $i(x), i^{*}(x)$ in any sphere $V$ (Theorem 3.3).

So far nothing prevents the points $x$ in which these extrema are taken from being on the boundary of $V$. If however one adds the generalized Hammerstein condition (3.38), an easy estimate shows that for a large enough $V$ the minimum of $i(x)$ can not be taken on the boundary. Thus the existence of a minimum taken in an interior point of $V$ is proved (Theorem 3.4).

In such a point $x$ the Fréchet differential $d(x, h)$ of $i(x)$ must be zero for all $h$. Therefore the Theorem 4.1 concerning the existence of a solution of the nonlinear integral equation system (4.6) follows nearly immediately sincespeaking crudely - the differential $d(x, h)$ can be written as a scalar product (cf. (4.7)) one factor of which is an "arbitrary" function $h^{*}(t)$ (corresponding to the arbitrary element $h$ ) and the other factor is the left member of the equations (4.6). In the special case of symmetric and definite kernels Theorem 4.1 yields immediately the Theorem 4.2 , a theorem proved first by Golomb [ 4 , $\$ 1$ ] under somewhat stricter hypotheses. Specialized to the case of one equation Theorem 4.2 contains the theorem about existence given by Hammerstein in $[6]\left({ }^{7}\right)$ as will be shown in $\$ 5$.

The generalizations by M. Golomb to the nonsymmetric case referred to in the first paragraph of this section deal with systems (4.9) without the assumptions of symmetry and definiteness (and also without the assumption (3.4) about the $f_{i}$ 's). The generalization to the nonsymmetric case treated in

(6) [5] and [6, §6]. In case that $K(s, t)$ is a Green's function (1.3) is the Dirichlet integral connected with the corresponding boundary value problem, cf. [6, p. 126].

${ }^{(7)}$ The theorems of [6] concerned only with existence problems for integral equations are the theorems $1,2,3$ of which 3 is a consequence of Theorem 2. 
the present paper, that is, the generalization from Theorem 4.2 to Theorem 4.1 , goes in another direction; it is of the same type as the generalization of the theory of the eigenfunctions of a linear integral equation with symmetric kernel to the Erhard Schmidt theory of the pairs of eigenfunctions of an unsymmetric kernel $[10, \S \S 13-17]$. This latter theory is extensively used in the present paper, especially in $\S 2$.

2. The auxiliary mappings $\Phi$ and $\Phi^{*}$. Let $E^{r}$ be the $r$-dimensional Euclidean space and let $s, t$ denote points of $E^{r}$. It is customary to call a kernel $K(s, t)$ admissible $\left(^{8}\right)$ if its eventual singularities are such that the ordinary theory of linear integral equations still holds. To make this somewhat loose terminology more precise we define for the purpose of this paper:

Definition 2.1. Domains $D$ of $E^{r}$ and kernels $K(s, t)$ defined for $s, t$ in $D$ are called admissible if they satisfy the conditions enumerated in $[10$, $\S 17]$.

Let then $D$ be an admissible domain and $K(s, t)$ an admissible kernel in $D$. We shall use a complete system of pairs of eigenfunctions $\phi_{\rho}(s), \phi_{\rho}^{*}(s)$ and eigenvalues $\lambda_{\rho}$ associated with $K(s, t)$. This system first introduced by $\mathrm{E}$. $\operatorname{Schmidt}\left({ }^{9}\right)$ has the following properties: the functions $\phi_{\rho}(s), \phi_{\rho}^{*}(s)$ are an at most countable $\left({ }^{10}\right)$ system of normed functions for which

$$
\begin{aligned}
\lambda_{\rho} \phi_{\rho}(s)= & \int_{D} K(s, t) \phi_{\rho}^{*}(t) d t, \quad \lambda_{\rho} \phi_{\rho}^{*}(s)=\int_{D} K(t, s) \phi_{\rho}(t) d t, & \\
& \int_{D} \phi_{\rho}(t) \phi_{\sigma}(t) d t=\int_{D} \phi_{\rho}^{*}(t) \phi_{\sigma}^{*}(t) d t=0 . & \rho \neq \sigma .
\end{aligned}
$$

The eigenvalues $\lambda_{\rho}$ satisfy the inequalities

$$
\sum_{\rho} \lambda_{\rho}^{2} \leqq \int_{D} \int_{D} K^{2}(s, t) d s d t
$$

and

$$
\lambda_{1} \geqq \lambda_{2} \geqq \lambda_{3} \geqq \cdots>0 ; \quad \lim _{\rho \rightarrow \infty} \lambda_{\rho}=0
$$

(8) "Brauchbar unstetig."

( 9$)[10, \S \$ 14,15]$. It should be noted that the terminology of the present paper differs from the one used by Schmidt and also by Hammerstein in that the eigenvalues $\lambda_{\rho}$ are replaced by $1 / \lambda_{\rho}$.

${ }^{(10)}$ In what follows the letter $\rho$ will always denote an arbitrary positive integer if there are infinitely many eigenfunctions and an integer between 1 and $N$ if there are only a finite number $N$ of eigenfunctions. Correspondingly the symbol $\sum_{\rho}$ stands for $\sum_{\rho=1}^{\infty}$ in the first case, and for $\sum_{\rho=1}^{N}$ in the second. In this latter case the symbol $\left(x_{1}, x_{2}, \cdots\right)$ is to mean $\left(x_{1}, x_{2}, \cdots, x_{N}\right)$ such that in the following definition $2.2, H=H_{1}$ is a finite-dimensional space. We shall not in each instance indicate the simplifications possible (like omission of convergence proofs) or the obvious modifications of the proofs necessary in the finite case. 
Definition 2.2. The Hilbert spaces $H$ and $H_{1}$ are defined in the following way: $H$ is the space of all sequences $x=\left(x_{1}, x_{2}, \cdots\right)$ of real numbers for which

$$
\sum_{\rho} \lambda_{\rho} x_{\rho}^{2}<\infty
$$

with the scalar product $(x, y)$ of $x$ with $y=\left(y_{1}, y_{2}, \cdots\right)$ and the norm $\|x\|$ of $x$ defined by

$$
(x, y)=\sum_{\rho} \lambda_{\rho} x_{\rho} y_{\rho} ; \quad\|x\|=+(x, x)^{1 / 2(11)} .
$$

$H_{1}$ is the subspace of $H$ consisting of those $x \subset H$ for which

$$
\sum_{\rho} x_{\rho}^{2}<\infty .
$$

Definition 2.3. $L_{2}$ is the Hilbert space of those functions $y(t)$ defined in $D$ for which $\int_{D} y^{2}(t) d t<\infty$ where the integral is taken in the sense of Lebesgue. $N$ is the subspace of those functions $n(t)$ of $L_{2}$ which are characterized by each of the following two equivalent conditions

$$
\int_{D} n(t) K(t, s) d t=0, \quad \int_{D} n(t) \phi_{\rho}(t) d t=0 \text { for all } \rho\left({ }^{12}\right) .
$$

$M$ is the orthogonal complement to $N$ in $L_{2}$, that is, the space spanned by the $\phi_{\rho}(s)$. The spaces obtained by substituting $\phi_{\rho}^{*}(s)$ for $\phi_{\rho}(s)$ and $K(s, t)$ for $K(t, s)$ in the definitions of $N$ and $M$ are called $N^{*}$ and $M^{*}$ respectively.

It follows easily from these definitions together with the Fischer-Riesz theorem that to each $y=\left(y_{1}, y_{2}, \cdots\right) \subset H_{1}$ there exists a uniquely determined $y(t) \subset M$ and a uniquely determined $y^{*}(t) \subset M^{*}$ such that for all $\rho$

$$
\begin{array}{ll}
y_{\rho}=\int_{D} y(t) \phi_{\rho}(t) d t, & \sum_{\rho} y_{\rho}^{2}=\|y(t)\|^{2}, \\
y_{\rho}=\int_{D} y^{*}(t) \phi_{\rho}^{*}(t) d t, & \sum_{\rho} y_{\rho}^{2}=\left\|y^{*}(t)\right\|^{2} .
\end{array}
$$

Definition 2.4. The obviously linear mappings which map the point $y$ of $H_{1}$ into $y(t) \subset M$ and $y^{*}(t) \subset M^{*}$ according to (2.8) and $\left(2.8^{*}\right)$ are called $\Phi_{1}$ and $\Phi_{1}^{*}$ respectively. $\Phi_{2}$ is the mapping which maps the point $x=\left(x_{1}, x_{2}, \cdots\right)$ $C H$ into the point $y=\left(y_{1}, y_{2}, \cdots\right)$ of $H_{1}$ with

$$
y_{\rho}=\lambda_{\rho} x_{\rho}\left({ }^{13}\right) \text {. }
$$

(11) It follows easily from (2.4) and the Schwarz inequality that the sum defining $(x, y)$ converges absolutely.

(12) For the equivalence of the two conditions see $[10, \$ 16]$.

(13) That $y \subset H_{1}$ follows from (2.3) and (2.4) since $\sum_{\rho} y_{\rho}^{2}=\sum_{\rho} \lambda_{\rho}^{2} x_{\rho}^{2} \leqq \lambda_{1} \sum_{\rho} \lambda_{\rho} x_{\rho}^{2}<\infty$. 
Finally the mappings $\Phi$ and $\Phi^{*}$ are defined as the obviously linear mappings $\Phi(x)=\Phi_{1}\left(\Phi_{2}(x)\right)$ and $\Phi^{*}(x)=\Phi_{1}^{*}\left(\Phi_{2}(x)\right)$. For fixed $x$ the two functions $y(t)=\phi(x)$, $y^{*}(t)=\Phi^{*}(x)$ are said to be conjugate.

LEMMA 2.1. The mappings $\phi$ and $\phi^{*}$ are bounded with the bound $\lambda_{1}^{1 / 2}$.

Proof.

$$
\|\phi(x)\|^{2}=\int_{D} y^{2}(t) d t=\sum_{\rho} y_{\rho}^{2}=\sum_{\rho} \lambda_{\rho}^{2} x_{\rho}^{2} \leqq \lambda_{1} \sum_{\rho} \lambda_{\rho} x_{\rho}^{2}=\lambda_{1}\|x\|^{2},
$$

and for $x=\left(x_{1}, 0,0, \cdots\right)$ we have the equality sign. The proof for $\Phi^{*}$ is analogous.

LeMma 2.2. The mappings $\Phi$ and $\Phi^{*}$ are completely continuous.

Proof. By Lemma 2.1 the mappings are continuous. To prove the complete continuity of $\Phi$ it will be sufficient [7, Lemma 5.2] to show: if $V$ is the sphere $\|x\| \leqq R$ there exists to each positive $\epsilon$ a finite-dimensional subspace $\bar{L}$ of $L_{2}$ and a mapping $\bar{\phi}(x)$ such that for $x \subset V$

(i) $\bar{\Phi}(x) \subset \bar{L}$,

(ii) $\|\Phi(x)-\bar{\Phi}(x)\|<\epsilon$.

To construct such an $\bar{L}$ and $\Phi$ we choose an integer $N$ such that $\lambda_{N+1}<\epsilon^{2} / R^{2}$ which is possible because of (2.3). We define $\bar{L}$ as the subspace of $L_{2}$ spanned by $\phi_{1}(s), \cdots, \phi_{N}(s)$ and set $\bar{\Phi}(x)=\sum_{i=1}^{N} \lambda_{i} x_{i} \phi_{i}(s)$. Then $\phi(x) \subset \bar{L}$ and

$$
\|\Phi(x)-\bar{\Phi}(x)\|^{2}=\sum_{i=N+1}^{\infty} \lambda_{i}^{2} x_{i}^{2} \leqq \lambda_{N+1} \sum_{i=N+1}^{\infty} \lambda_{i} x_{i}^{2} \leqq \lambda_{N+1}\|x\|^{2} \leqq \lambda_{N+1} R^{2}
$$

which because of the choice of $N$ proves (ii). The proof for $\Phi^{*}$ is analogous.

LEMMA 2.3. For $x \subset H_{1}$, let $y(t)=\Phi(x), y^{*}(t)=\Phi^{*}(x)$ and $x(t)=\Phi_{1}(x), x^{*}(t)$ $=\Phi_{1}^{*}(x)$. Then

$$
y(t)=\int_{D} K(t, s) x^{*}(s) d s, \quad y^{*}(t)=\int_{D} K(s, t) x(s) d s .
$$

Proof. By (2.9), (2.8*), by the definition of $\Phi$ and by (2.1) we obtain

$$
\begin{aligned}
y_{\rho}=\lambda_{\rho} x_{\rho} & =\lambda_{\rho} \int_{D} x^{*}(s) \phi_{\rho}^{*}(s) d s=\int_{D} x^{*}(s) \int_{D} K(t, s) \phi_{\rho}(t) d t d s \\
& =\int_{D} \phi_{\rho}(t)\left\{\int_{D} K(t, s) x^{*}(s) d s\right\} d t .
\end{aligned}
$$

This shows that like $y(t)=\Phi(x)$ the function 


$$
\bar{y}(t)=\int_{D} K(t, s) x^{*}(s) d s
$$

satisfies the equations (2.8). Therefore we shall have proved $y(t)=\bar{y}(t)$, that is, the first equation (2.10), once we have shown that both functions are in the subspace $M$ of $L_{2}$. But $y(t) \subset M$ by definition, and that also $\bar{y}(t) \subset M$ is seen immediately from the fact that by the Schmidt expansion theorem $[10$, p. 465] this function can be developed according to the eigenfunctions $\phi_{\rho}(s)$. This proves the first equation (2.10) and the second one is proved correspondingly.

3. The Hammerstein scalars $i(x)$ and $i^{*}(x)$. Let $D_{0}$ be an admissible domain of the $r$-dimensional Euclidean space $E^{r}$, and $K_{i j}(s, t)(i, j$ $=1,2, \cdots, n) n$ admissible kernels in $D_{0}$. We choose a point $d_{0}$ in $E^{r}$ of the following property: if $D_{i}$ denotes the domain of all points $\left({ }^{14}\right) s+i d_{0}$ where $s$ ranges over $D_{0}$, then the domains $D_{0}, D_{1}, \cdots, D_{n-1}$ are mutually disjoint. The union $D$ of these domains is an admissible domain and by setting

$$
\begin{aligned}
& K(s, t)=K_{i+1, j+1}\left(s-i d_{0}, t-j d_{0}\right) \quad \text { for } \quad s \subset D_{i}, t \subset D_{i} \\
& \\
& \qquad(i, j=0,1, \cdots, n-1)
\end{aligned}
$$

we obtain an admissible kernel in $D$. Correspondingly, for any ordered $n$-tuple of functions $y_{1}(s), y_{2}(s), \cdots, y_{n}(s)$ with domain $D_{0}$, we define a function $y(s)$ with domain $D$ by setting

$$
y(s)=y_{i+1}\left(s-i d_{0}\right) \quad \text { for } \quad s \subset D_{i} \quad(i=0,1, \cdots, n-1) .
$$

Conversely, a given function $y(s)$ with domain $D$ defines by (3.2) uniquely an ordered $n$-tuple of functions $y_{1}(s), y_{2}(s), \cdots, y_{n}(s)$ with domain $D_{0}$. The two $n$-tuples $y_{1}(s), \cdots, y_{n}(s)$, and $y_{1}^{*}(s), \cdots, y_{n}^{*}(s)$ are called conjugate if the corresponding functions $y(s), y^{*}(s)$ are conjugate (see Definition 2.4).

We identify the domain $D$ and the kernel $K(s, t)$ just defined with the domain $D$ and the kernel $K(s, t)$ of $\$ 2$. For later use we note the following inequality: if $h=\left(h_{1}, h_{2}, \cdots\right)$ is an element of the space $H$ (Definition 2.2) and if $k(s)=\Phi(h)$ (Definition 2.4), then

$$
\int_{D_{0}} k_{j}^{2}(t) d t \leqq \sum_{i=1}^{n} \int_{D_{0}} k_{i}^{2}(t) d t=\|k(t)\|^{2} \leqq \lambda_{1}\|h\|^{2} \quad(j=1,2, \cdots, n) .
$$

Indeed one sees easily from (3.2) that

$$
\sum_{i=0}^{n} \int_{D_{0}} k_{i}^{2}(t) d t=\int_{D} k^{2}(s) d s=\|k(s)\|^{2}
$$

which on account of Lemma 2.1 proves (3.3).

${ }^{(14)} s+t$ denotes the point of $E^{r}$ whose coordinates are the sum of the coordinates of $s$ and $t$. 
Let now $f_{i}\left(t, u_{1}, u_{2}, \cdots, u_{n}\right)(i=1,2, \cdots, n)$ be $n$ functions defined and continuous in all points of the product space whose points are the pairs $(t, U)$ where $t$ denotes a point of $D_{0}$ and $U$ the point with coordinates $u_{1}, u_{2}, \cdots, u_{n}$ of the Euclidean $n$-space $E^{n}$. We suppose that $\sum_{i=1}^{n} f_{i} d u_{i}$ is a total differential, in other words that the line integral

$$
F(t, U)=\int_{0}^{U} \sum_{i=1}^{n} f_{i}\left(t, u_{1}, u_{2}, \cdots, u_{n}\right) d u_{i}
$$

is a function of $t$ and $U$ alone.

We then define the Hammerstein scalars $i(x)$ and $i^{*}(x)$ in the following way:

Definition 3.1. Let $x$ be an arbitrary point of the space $H$ (Definition 2.2). Let $y(t)=\Phi(x), y^{*}(t)=\Phi^{*}(x)$ (Definition 2.4), and let, for each $t \subset D_{0}$, $Y(t), Y^{*}(t)$ be those points of $E^{n}$ whose coordinates are the values of the $n$-tuples of functions $y_{i}(t), y_{i}^{*}(t)(i=1,2, \cdots, n)$ associated with the functions $y(t), y^{*}(t)$ respectively by means of the rule (3.2). We then set

$$
\begin{aligned}
I(x) & =\int_{D_{0}} F(t, Y(t)) d t, & I^{*}(x) & =\int_{D_{0}} F\left(t, Y^{*}(t)\right) d t, \\
i(x) & =\|x\|^{2} / 2+I(x), & i^{*}(x) & =\|x\|^{2} / 2+I^{*}(x) .
\end{aligned}
$$

The integrals (3.5) will certainly exist under the conditions of Theorem 3.1. This is obvious for condition (B); for condition (A) it follows from Lemma 3.1.

THEOREM 3.1. Let the functions $f_{i}\left(t, u_{1}, u_{2}, \cdots, u_{n}\right)$ satisfy the conditions siated above and let the kernels $K_{i j}(s, t)(i, j=1,2, \cdots, n)$ be admissible in the domain $D_{0}$. Let $y(t), y^{*}(t), y_{i}(t), y_{i}^{*}(t)$ have the same meaning as in Definition 3.1. Finally let the functions $k_{i}(t), k_{i}{ }^{*}(t)$ be derived from the element $h$ of $H$ in the same way as the functions $y_{i}(t), y_{i}^{*}(t)$ are derived from the element $x$ of $H$. then: by

(i) in order that $I(x)$ have a continuous $\left({ }^{15}\right)$ Fréchet differential $D(x, h)$ given

$$
D(x, h)=\sum_{i=1}^{n} \int_{D_{0}} k_{i}(t) f_{i}\left(t, y_{1}(t), \cdots, y_{n}(t)\right) d t
$$

each of the following two conditions (A) and (B) is sufficient:

(A) there exists a constant $C$ independent of $s$ such that

(15) The terms continuity, complete continuity applied to $D(x, h)$ are meant in the sense of the definitions given in $[9, \S 3]$, that is, $D(x, h)$ is considered as a mapping which maps the point $x \subset H$ into the point $l_{x}(h)=D(x, h)$ of the space $H^{*}$ conjugate to $H$, and the terms continuity, complete continuity refer to the corresponding properties of this mapping. The corresponding remark holds for $D^{*}(x, h)$. 


$$
\sum_{\rho} \lambda_{\rho} \phi_{\rho}^{2}(s) \leqq C^{2} \quad \text { for all } s \subset D_{0}\left({ }^{16}\right) .
$$

(B) there exist constants $a$ and $b$, both independent of $s$ and the $u_{i}$, such that for all $s \subset D_{0}$ and all $u_{i}\left(-\infty<u_{i}<+\infty ; i=1,2, \cdots, n\right)$,

$$
\left|f_{i}\left(s, u_{1}, u_{2}, \cdots, u_{n}\right)\right|<a+b\left(\left|u_{1}\right|+\left|u_{2}\right|+\cdots+\left|u_{n}\right|\right) \text {. }
$$

(ii) in order that $I^{*}(x)$ have a continuous Frêchet differential $D^{*}(x, h)$ given by

$$
D^{*}(x, h)=\sum_{i=1}^{n} \int_{D_{0}} k_{i}^{*}(t) f_{i}\left(t, y_{1}^{*}(t), \cdots, y_{n}^{*}(t)\right) d t
$$

each of the conditions $\left(\mathrm{A}^{*}\right)$ and $(\mathrm{B})$ is sufficient where $\left(\mathrm{A}^{*}\right)$ is obtained by substituting $\phi_{\rho}^{*}(s)$ for $\phi_{p}(s)$ in the condition $(\mathrm{A})$.

Proof. It will be sufficient to prove (i). Because of the condition (3.4) and the linearity of the mapping $\Phi$ we have

$$
\begin{array}{r}
I(x+h)-I(x)=\int_{D_{0}}\left\{\int_{Y(t)}^{Y(t)+K(t)} \sum_{i=1}^{n} f_{i}\left(t, u_{1}, u_{2}, \cdots, u_{n}\right) d u_{i}\right\} d t \\
=\int_{D_{0}} \int_{0}^{1} \sum_{i=1}^{n} k_{i}(t) f_{i}\left(t, y_{1}(t)+\theta k_{1}(t), \cdots, y_{n}(t)\right. \\
\left.+\theta k_{n}(t)\right) d \theta d t
\end{array}
$$

where $Y(t)$ has the same meaning as in Definition 3.1 and correspondingly $K(t)$ denotes the point of $E^{n}$ with coordinates $k_{1}(t), \cdots, k_{n}(t)$. If $D(x, h)$ is given by (3.6) and if

$$
\begin{array}{r}
R(x, h, \theta)=\int_{D_{0}} \sum_{i=1}^{n} k_{i}(t)\left\{f _ { i } \left(t, y_{1}(t)+\theta k_{1}(t), \cdots, y_{n}(t)+\theta k_{n}(t)\right.\right. \\
\left.-f_{i}\left(t, y_{1}(t), \cdots, y_{n}(t)\right)\right\} d t
\end{array}
$$

the use of the mean value theorem of the integral calculus shows that (3.9) may be written in the form

$$
I(x+h)-I(x)=D(x, h)+R\left(x, h, \theta^{*}\right)
$$

for some $\theta^{*}$ between 0 and 1 . Clearly the theorem will be proved if we can show: to given positive $\epsilon$ there exists a positive number $h_{0}$ such that

$$
|R(x, h, \theta)|<\epsilon\|h\|
$$

(16) Obviously (A) will be satisfied whenever the so-called bilinear series $\sum_{\rho} \lambda_{\rho} \phi_{\rho}(s) \phi_{\rho}(t)$ converges uniformly. A number of sufficient conditions for this to be the case are known in the case of a symmetric kernel $K(s, t)$. If $K(s, t)$ is positive definite and symmetric it will be shown in $\$ 5$ that (A) is certainly satisfied whenever $K(s, t)$ is in addition bounded and admissible. 
provided that the real number $\theta$ and the element $h$ of $H$ satisfy the inequalities

$$
0 \leqq \theta \leqq 1, \quad\|h\| \leqq h_{0} .
$$

We take up first the case that assumption (A) is satisfied and state the following lemma.

LEMMA 3.1. Under the assumption (A) the function $y(t)=\Phi(x)$ (Definition 2.4) is a continuous function of $t$. Moreover

$$
|y(t)| \leqq\|x\| \cdot C \text {. }
$$

Proof. Let $x=\left(x_{1}, x_{2}, \cdots\right)$ and $y_{p}=\lambda_{\rho} x_{\rho}$. It is easily seen from the definition of the mapping $\Phi$ that if the sum

$$
\sum_{\rho} y_{\rho} \phi_{\rho}(s)
$$

converges uniformly in $D_{0}$ then this sum will be $y(t)$, and then $y(t)$ will be of course continuous. To prove the uniform convergence of (3.15) we note that for any two integers $p<q$ by virtue of the Schwarz inequality and the assumption (A)

$$
\begin{aligned}
\left|\sum_{i=p}^{q} y_{i} \phi_{i}(s)\right| & \leqq \sum_{i=p}^{q}\left|y_{i} \phi_{i}(s)\right|=\sum_{i=p}^{q}\left|\lambda_{i} x_{i} \phi_{i}(s)\right| \\
& \leqq\left(\sum_{i=p}^{q} \lambda_{i} x_{i}^{2}\right)^{1 / 2}\left(\sum_{i=p}^{q} \lambda_{i} \phi_{i}^{2}(s)\right)^{1 / 2} \leqq C\left(\sum_{i=p}^{q} \lambda_{i} x_{i}^{2}\right)^{1 / 2} .
\end{aligned}
$$

Because of (2.4) this proves the uniform convergence of the sum (3.15). At the same time since this sum equals $y(t)$ we obtain also (3.14) from (2.5) and (3.16) by letting $p=1$ and $q$ approach $\infty$.

We continue with the proof of (3.12) and denote for any measurable subset $e$ of $D_{0}$ the measure of $e$ by $\mu(e)$. Since the functions $f_{i}(t)$ are continuous, there exists to the given $\epsilon$ an $\eta_{0}>0$ such that

$$
\begin{array}{r}
\mid f_{i}\left(t, y_{1}(t)+\eta_{1}, \cdots, y_{n}(t)+\eta_{n}\right)-f_{i}\left(t, y_{1}(t), \cdots, y_{n}(t) \mid<\frac{\epsilon}{n C \mu\left(D_{0}\right)}\right. \\
\text { for }\left|\eta_{j}\right|<\eta_{0}(i, j=1,2, \cdots, n) .
\end{array}
$$

Since on the other hand by Lemma $3.1\left|k_{i}(t)\right| \leqq C\|h\|$, we have for $0 \leqq \theta \leqq 1$

$$
\left|\theta k_{i}(t)\right| \leqq\left|k_{i}(t)\right| \leqq C\|h\| \leqq \eta_{0} \quad \text { for }\|h\| \leqq \eta_{0} / C .
$$

Using this we see easily from (3.10) and (3.17) that (3.12) is true if only (3.13) is satisfied with $h_{0}=\eta_{0} / C$. This finishes the proof of Theorem 3.1 in case of the assumption (A).

We turn now to the more complicated case of assumption (B) and prove 
first the following lemma.

LEMMA 3.2. Let $y_{i}(t)$ and $k_{i}(t)$ have the same meaning as in the statement of Theorem 3.1. Let e be a measurable subset of $D_{0}$ and as usual let $\mu(e)$ be the measure of $e$. Under the assumption (B) we have then for $0 \leqq \theta \leqq 1$ :

$$
\begin{aligned}
& \mid \sum_{i=1}^{n} \int_{e} k_{i}(t)\left\{f_{i}\left(t, y_{1}+\theta k_{1}(t), \cdots, y_{n}(t)+\theta k_{n}(t)\right)\right. \\
& \left.-f_{i}\left(t, y_{1}(t), \cdots, y_{n}(t)\right)\right\} d t \\
& \leqq\left\{2 a(\mu(e))^{1 / 2}+2 b \sum_{j=1}^{n}\left(\int_{e} y_{j}^{2}(t) d t\right)^{1 / 2}+b \cdot n \lambda_{1}^{1 / 2}\|h\|\right\} \cdot n \lambda_{1}^{1 / 2}\|h\| .
\end{aligned}
$$

Proof. By the use of the Schwarz inequality and of the assumption (B) we see that the left member of (3.18) is not greater than

$$
\begin{aligned}
\sum_{i=1}^{n} \int_{e}\left|k_{i}(t)\right|\{2 a+ & \left.2 b \cdot \sum_{j=1}^{n}\left|y_{j}(t)\right|+\theta b \sum_{j=1}^{n}\left|k_{j}(t)\right|\right\} d t \\
\leqq 2 a \sum_{i=1}^{n}(\mu(e))^{1 / 2}\left(\int_{e} k_{i}^{2}(t) d t\right)^{1 / 2} & \\
& +2 b \sum_{i=1}^{n} \sum_{j=1}^{n}\left(\int_{e} y_{j}^{2}(t) d t\right)^{1 / 2}\left(\int_{e} k_{i}^{2}(t) d t\right)^{1 / 2} \\
& +b \sum_{i=1}^{n} \sum_{j=1}^{n}\left(\int_{e} k_{i}^{2}(t) d t\right)^{1 / 2}\left(\int_{e}^{2}(t) d t\right)^{1 / 2}
\end{aligned}
$$

Since by (3.3)

$$
\sum_{i=1}^{n}\left(\int_{e} k_{i}^{2}(t) d t\right)^{1 / 2} \leqq \sum_{i=1}^{n}\left(\int_{D_{0}} k_{i}^{2}(t) d t\right)^{1 / 2} \leqq n \lambda_{1}^{1 / 2}\|h\|,
$$

(3.18) follows.

We resume the proof of (3.12). Let $\epsilon>0$ be given and

$$
\alpha=\sum_{i=1}^{n} \int_{D_{0}} y_{i}^{2}(t) d t
$$

Since

$$
\int_{e}^{y_{i}^{2}(t) d t}
$$

tends to zero as $\mu(e)$ approaches zero, there exists a $\bar{\mu}_{1}>0$ such that 


$$
\sum_{i=1}^{n} \int_{e} y_{i}^{2}(t) d t<\frac{1}{\lambda_{1}}\left(\frac{\epsilon}{18 n b}\right)^{2} \quad \text { if } \mu(e) \leqq \bar{\mu}_{1} .
$$

We set

$$
\mu_{1}=\min \left\{\bar{\mu}_{1}, \frac{1}{\lambda_{1}}\left(\frac{\epsilon}{18 a n}\right)^{2}\right\}
$$

and

$$
M=2\left(\alpha / \mu_{1}\right)^{1 / 2} .
$$

We now split $D_{0}$ into $D_{0}=e^{\prime}+e^{\prime \prime}$ such that

$$
\sum_{i=1}^{n} y_{i}^{2}(t)\left\{\begin{array}{l}
>(M / 2)^{2} \\
\leqq(M / 2)^{2}
\end{array}\right.
$$

Then by (3.19)

$$
\mu\left(e^{\prime}\right)(M / 2)^{2} \leqq \sum_{i=1}^{n} \int_{e^{\prime}} y_{i}^{2}(t) d t=\alpha ;
$$

therefore by (3.22)

$$
\mu\left(e^{\prime}\right) \leqq \mu_{1},
$$

and by (3.20) with $e=e^{\prime}$

$$
\sum_{i=1}^{n} \int_{\epsilon^{\prime}} y_{i}^{2}(t) d t \leqq \frac{1}{\lambda_{1}}\left(\frac{\epsilon}{18 n b}\right)^{2}
$$

We now write

$$
R(x, h, \theta)=R^{\prime}+R^{\prime \prime}
$$

where $R^{\prime}$ and $R^{\prime \prime}$ are the integrals with the integrand of (3.10) extended over $e^{\prime}$ and $e^{\prime \prime}$ respectively. Applying Lemma 3.2 with $e=e^{\prime}$ we obtain from (3.18), (3.24), (3.21) and (3.25)

$$
\left|R^{\prime}\right| \leqq\left\{2 \epsilon / 9+b n^{2} \lambda_{1}\|h\|\right\}\|h\|,
$$

that is, we shall have

$$
\left|R^{\prime}\right| \leqq \epsilon\|h\| / 3
$$

if only

$$
\|h\| \leqq \frac{\epsilon}{9 b n^{2} \lambda_{1}}=h_{0}^{\prime} .
$$

To estimate $R^{\prime \prime}$ we note that in $e^{\prime \prime}$ the $y_{i}(t)$ are bounded by $M / 2$. Since the $f_{i}\left(t, u_{1} \cdots u_{n}\right)$ are uniformly continuous if the $u_{i}$ 's are bounded it fol- 
lows that there exists an $\eta_{0}>0$ independent of $t$ such that for $t<e^{\prime \prime}$

$$
\left|f_{i}\left(t, y_{1}(t)+\eta_{1}, \cdots, y_{n}(t)+\eta_{n}\right)-f\left(t, y_{1}(t), \cdots, y_{n}(t)\right)\right|
$$

$$
<\frac{\epsilon}{3 n\left(\lambda_{1} \mu\left(D_{0}\right)\right)^{1 / 2}} \quad(i=1,2, \cdots, n)
$$

if

$$
\left|\eta_{j}\right|<\eta_{0} \quad(j=1,2, \cdots, n) .
$$

We now write $e^{\prime \prime}=e_{1}^{\prime \prime}+e_{2}^{\prime \prime}$ where $e_{1}^{\prime \prime}$ and $e_{2}^{\prime \prime}$ are such that

$$
\sum_{i=1}^{n} k_{i}^{2}(t) \begin{cases}<\eta_{0}^{2} & \text { for } t \subset e_{1}{ }^{2}, \\ \geqq \eta_{0}^{2} & \text { for } t \subset e_{2}^{\prime \prime} .\end{cases}
$$

Correspondingly we write

$$
R^{\prime \prime}=R_{1}^{\prime \prime}+R_{2}^{\prime \prime}
$$

where $R_{1}^{\prime \prime}$ and $R_{2}^{\prime \prime}$ are the integrals with the integrand of (3.10) extended over $e_{1}^{\prime \prime}$ and $e_{2}^{\prime \prime}$ respectively.

We estimate first $R_{1}^{\prime \prime}$. If we set $\eta_{j}=\theta k_{i}(t)$ with $0 \leqq \theta \leqq 1$ the inequality (3.30) is satisfied for $t \subset e_{1}^{\prime \prime}$ and we obtain from (3.29) and (3.10)

$$
\begin{aligned}
\left|R_{1}\right| & \leqq \frac{\epsilon}{3 n\left(\lambda_{1} \mu\left(D_{0}\right)\right)^{1 / 2}} \sum_{i=1}^{n} \int_{e_{1}^{\prime \prime}}\left|k_{i}(t)\right| d t \\
& \leqq \frac{\epsilon}{3 n\left(\lambda_{1} \mu\left(D_{0}\right)\right)^{1 / 2}} \sum_{i=1}^{n}\left(\int_{e_{1}^{\prime \prime}} k_{i}^{2}(t) d t\right)^{1 / 2}\left(\mu\left(e_{1}^{\prime}\right)\right)^{1 / 2}
\end{aligned}
$$

Since $e_{1}^{\prime \prime} \subset D_{0}$ we see from (3.3) that a fortiori

$$
\left|R_{1}^{\prime \prime}\right| \leqq\|h\|_{\epsilon} / 3 \text {. }
$$

It remains to estimate $R_{2}^{\prime \prime}$. From (3:31) and (3.3) we see that

$$
\begin{aligned}
\underset{\eta_{0} \mu}{2}\left(e_{2}^{\prime \prime}\right) & \leqq \sum_{i=1}^{n} \int_{e_{2}^{\prime \prime}} k_{i}^{2}(t) d t \leqq \lambda_{1}\|h\|^{2}, \\
\mu\left(e_{2}^{\prime \prime}\right) & \leqq\|h\|^{2} \lambda_{1} / \eta_{0}^{2},
\end{aligned}
$$

and from (3.23) since $e_{2}^{\prime \prime} \subset e^{\prime \prime}$

$$
\int_{e_{2}^{\prime \prime}} y_{i}^{2}(t) d t \leqq(M / 2)^{2} \mu\left(e_{2}^{\prime \prime}\right) \leqq \lambda_{1}\left(\|h\| M / 2 \eta_{0}\right)^{2} .
$$

Applying Lemma 3.2 with $e=e_{2}^{\prime \prime}$ we obtain easily from (3.18), (3.34) and (3.35)

$$
\left|R_{2}^{\prime \prime}\right| \leqq\left(2 a / \eta_{0}+b M n / \eta_{0}+b n\right) \lambda_{1}\|h\|^{2}
$$


that is,

$$
\left|R_{2}^{\prime \prime}\right|<\|h\| \epsilon / 3
$$

if only

$$
\|h\| \leqq \frac{\epsilon \eta_{0}}{3 \lambda_{1}\left(2 a+b M n+b n \eta_{0}\right)}=h_{0}^{\prime \prime} .
$$

Since $R(x, h, \theta) \leqq\left|R^{\prime}\right|+\left|R_{1}^{\prime \prime}\right|+\left|R_{2}^{\prime \prime}{ }_{i}\right|$ we see by adding (3.27), (3.33) and (3.37) that (3.12) is true whenever (3.13) is satisfied with $h_{0}=\min \left(h_{0}^{\prime}, h_{0}^{\prime \prime}\right)$ where $h_{0}^{\prime}, h_{0}^{\prime \prime}$ are defined in (3.28), (3.37) respectively.

ThEOREM 3.2. Under the same assumptions as in Theorem 3.1 the differentials $D(x, h)$ and $D^{*}(x, h)$ are completely continuous $\left({ }^{17}\right)$.

Proof. The mapping which maps the point $x$ of the space $H$ into the point $l_{x}(h)=D(x, h)$ of the space $H^{*}$ of linear functionals $l(h)$ is the product of the mapping $\Phi$ which maps $x \subset H$ into $y(t) \subset L_{2}$ and the mapping which maps $y(t)$ into $l_{x}(h)=D(x, h)$ given by (3.6). The latter is continuous as an examination of the proof of Theorem 3.1 immediately shows, and the mapping $\Phi$ is completely continuous by Lemma 2.2. The product mapping is therefore completely continuous which proves the theorem for the differential $D(x, h)$. The proof for $D^{*}(x, h)$ is analogous.

TheOREM 3.3. Let $V_{R}$ denote the sphere $\|x\| \leqq R$ of the space $H$. Under the assumptions of Theorem 3.1 the scalars $I(x)$ and $I^{*}(x)$ (Definition 3.1) take a maximum and a minimum in $V_{R}$. The Hammerstein scalars $i(x)$ and $i^{*}(x)$ take a minimum in $V_{R}$.

Proof. Because of the Theorems 3.1 and 3.2 the assertion is an immediate consequence of Theorem 1.1 since $H$ is a reflexive Banach space.

THEOREM 3.4. In addition to the assumptions of Theorem 3.1 we suppose that

$$
F(t, U) \geqq-\frac{k}{2} \sum_{i=1}^{n} u_{i}^{2}-C_{1}
$$

where $F(t, U)$ is the function defined in (3.4) and where $C_{1}$ and $k$ are constants, the latter subject to the condition

$$
0 \leqq k<1 / \lambda_{1}
$$

Then there exists an $R_{0}$ such that for $R>R_{0}$ the Hammerstein scalars $i(x), i^{*}(x)$ take a minimum in an interior point of the sphere $V_{R}$.

Proof. We prove the theorem for $i(x)$. Since by Theorem 3.3 a minimum

${ }^{(17)} \mathrm{Cf}$. footnote 15 . 
is taken in any sphere $V_{R}$, we have only to prove that for large enough $R$ the minimum cannot be taken on the boundary of $V_{R}$. In fact we shall show: if

$$
R_{0}^{2}=2 \frac{I(0)+C_{1} \mu\left(D_{0}\right)}{1-k \lambda_{1}}
$$

then

$$
i(x)>i(0)
$$$$
\text { for }\|x\|>R_{0} \text {. }
$$

We have indeed from (3.4), (3.5) and (3.38)

$$
\begin{aligned}
I(x) & \geqq-\int_{D_{0}}\left[\frac{k}{2} \sum_{i=1}^{n} y_{i}^{2}(t)+C_{1}\right] d t=-\frac{k}{2} \sum_{\rho} y_{\rho}^{2}-C_{1} \mu\left(D_{0}\right) \\
& =-\frac{k}{2} \sum_{\rho} \lambda_{\rho}^{2} x_{\rho}^{2}-C_{1} \mu\left(D_{0}\right) \geqq-\frac{k}{2} \lambda_{1} \sum_{\rho} \lambda_{\rho} x_{\rho}^{2}-C_{1} \mu\left(D_{0}\right) .
\end{aligned}
$$

Therefore by (2.5)

$$
i(x)=\|x\|^{2} / 2+I(x) \geqq\left(\|x\|^{2} / 2\right)\left(1-k \lambda_{1}\right)-C_{1} \mu\left(D_{0}\right) .
$$

Since $1-k \lambda_{1}>0$ by (3.39), we obtain

$$
i(x)>R_{0}^{2}\left(1-k \lambda_{1}\right) / 2-C_{1} \mu\left(D_{0}\right) \quad \text { for }\|x\|>R_{0} .
$$

Substituting the value (3.40) of $R_{0}^{2}$ yields (3.41) since $I(0)=i(0)$.

\section{Application to nonlinear integral equations.}

LEMMA 4.1. If $h$ is an element of the subspace $H_{1}$ of $H$ (Definition 2.2) and if $h(t)=\Phi_{1}(h), h^{*}(t)=\phi_{1}^{*}(h)$ (Definition 2.4), then the differentials $D(x, h)$ and $D^{*}(x, h)$ of Theorem 3.1 may be written in the form

$$
\begin{aligned}
& \text { (4.1) } D(x, h)=\int_{D_{0}} \sum_{j=1}^{n} h_{j}^{*}(s)\left\{\int_{D_{0}} \sum_{i=1}^{n} K_{i j}(t, s) f_{i}\left(t, y_{1}(t), \cdots, y_{n}(t)\right) d t\right\} d s \\
& \text { (4.2) } D^{*}(x, h)=\int \sum_{D_{0}} \sum_{j=1}^{n} h_{j}(s)\left\{\int_{D_{0}} \sum_{i=1}^{n} K_{j i}(s, t) f_{i}\left(t, y_{1}^{*}(t), \cdots, y_{n}^{*}(t)\right) d t\right\} d s .
\end{aligned}
$$

Proof. From (3.1), (3.2) and Lemma 2.3 we obtain easily

$$
k_{i}(t)=\int_{D_{0}} \sum_{j=1}^{n} K_{i j}(t, s) h_{j}^{*}(s) d s \quad(i=1,2, \cdots, n) .
$$

Substituting this expression for $k_{i}(t)$ in the Definition (3.6) of $D(x, h)$ yields $(4.1) ;(4.2)$ is proved similarly.

LEMmA 4.2. Let $x=\left(x_{1}, x_{2}, \cdots\right)$ and $h=\left(h_{1}, h_{2}, \cdots\right)$ be arbitrary elements of $H$, and $y=\left(\lambda_{1} x_{1}, \lambda_{2} x_{2}, \cdots\right)$. Let $y(t)=\Phi_{1}(y)=\Phi(x)$ and $y^{*}(t)=\Phi_{1}^{*}(y)=\Phi^{*}(x)$. 
Finally set

$$
I_{1}(x)=\frac{1}{2} \sum_{\rho} \lambda_{\rho} x_{\rho}^{2}=\frac{1}{2}\|x\|^{2} .
$$

Then $I_{1}(x)$ has a Fréchet differential $D_{1}(x, h)$ given by

$$
D_{1}(x, h)=\sum_{\rho} \lambda_{\rho} x_{\rho} h_{\rho}
$$

If, especially, $h \subset H_{1}$, the differential $D_{1}(x, h)$ may be written as

$$
D_{1}(x, h)=\sum_{j=1}^{n} \int_{D_{0}} y_{j}(s) h_{j}(s) d s=\sum_{j=1}^{n} \int_{D_{0}} y_{j}^{*}(s) h_{j}^{*}(s) d s
$$

where $h(s)=\Phi_{1}(h), h^{*}(s)=\phi_{1}^{*}(h)$ and where the $n$-tuples $y_{j}(s), y_{j}^{*}(s), h_{j}(s)$, $h_{j}^{*}(s)$ are associated with the functions $y(s), y^{*}(s), h(s), h^{*}(s)$ respectively by means of the rule (3.2).

Proof. (4.4) follows from the identity

$$
I_{1}(x+h)-I_{1}(x)=\sum_{\rho} \lambda_{\rho} x_{\rho} h_{\rho}+\frac{1}{2}\|h\|^{2} .
$$

Moreover we obtain from (4.4), (2.8) and (3.2)

$$
D_{1}(x, h)=\sum_{\rho} h_{\rho} y_{\rho}=\int_{D} h(t) y(t) d t=\sum_{i=1}^{n} \int_{D_{0}} h_{i}(t) y_{i}(t) d t
$$

which is the first part of (4.5). The second part follows the same way.

THEOREM 4.1. Under the assumptions of Theorem 3.4 the following two systems of nonlinear integral equations for the conjugate n-tuples (see Definition 2.4 and the end of the first paragraph of $\$ 3) y_{j}(s), y_{j}^{*}(s)$ and $\bar{y}_{j}(s), \bar{y}_{j}^{*}(s)$ $(j=1,2, \cdots, n)$ have at least one solution

$$
\begin{gathered}
y_{j}^{*}(s)+\int_{D_{0}} \sum_{i=1}^{n} K_{i j}(t, s) f_{i}\left(t, y_{1}(t), \cdots, y_{n}(t)\right) d t=0, \\
\bar{y}_{j}(s)+\int_{D_{0}} \sum_{i=1}^{n} K_{j i}(s, t) f_{i}\left(t, \bar{y}_{1}^{*}(t), \cdots, \bar{y}_{n}^{*}(t)\right) d t=0 .
\end{gathered}
$$

Proof. By Theorem 3.4 the scalar $i(x)=\|x\|^{2} / 2+I(x)$ takes a minimum in some interior point of the sphere $V_{R}$ if $R$ is large enough. In such a point $x$ the Fréchet differential $D_{1}(x, h)+D(x, h)$ of $i(x)$ must be zero for all $h \subset H\left({ }^{18}\right)$. We apply this especially to elements $h$ of the subspace $H_{1}$ of $H$. For such $h$ we may use Lemmas 4.1 and 4.2 and write the equation $D_{1}(x, h)+D(x, h)=0$

(18) That the Fréchet differential of a scalar must vanish in an interior extremum point is easy to see. For a proof see, for example, [8, Lemma 2.4 and remark to Lemma 2.4]. 
in the form

$$
\int_{D_{0}} \sum_{j=1}^{n} h_{j}^{*}(s)\left[y_{j}^{*}(s)+\int_{D_{0}} \sum_{i=1}^{n} K_{i j}(t, s) f_{i}\left(t, y_{1}(t), \cdots, y_{n}(t)\right) d t\right] d s=0,
$$

where $y_{j}(s), y_{j}^{*}(s)$ are the $n$-tuples of functions associated with $y(s)=\Phi(x)$, $y^{*}(s)=\Phi^{*}(x)$ respectively by the rule (3.2). We set for a moment $f_{i}(t)$ $=f_{i}\left(t, y_{1}(t), \cdots, y_{n}(t)\right)$. By the use of (3.1), (3.2) equation (4.7) may then be written in the form

$$
\int_{D} h^{*}(s)\left[y^{*}(s)+\int_{D} K(t, s) f(t) d t\right] d s=0 .
$$

This shows that [ ] is orthogonal to $h^{*}(s)=\Phi_{1}^{*}(h)$ for all $h \subset H_{1}$, therefore especially to all $\phi_{\rho}^{*}(s)\left({ }^{19}\right)$. Since these functions span $M^{*}$ we can conclude []$=0$ once we know that [ $] \subset M^{*}$. But this is true since $y^{*}(s) \subset M^{*}$ by definition and since the integral in [ ] can be expanded according to the $\phi_{\rho}^{*}(s)$ by the Schmidt expansion theorem [10, p. 465]. This shows that the first set of equations (4.6) is satisfied since the left member of these equations is the expression [ ]. The validity of the second set of (4.6) is proved correspondingly.

We consider now the symmetric and positive definite case. The system

$$
y_{j}(s)+\int_{D_{0}} \sum_{i=1}^{n} K_{j i}(s, t) f_{i}\left(t, y_{1}(t), \cdots, y_{n}(t)\right) d t=0 \quad(j=1,2, \cdots, n)
$$

is called symmetric if the kernel $K(s, t)$ defined by (3.1) is symmetric; it is called positive definite if $K(s, t)$ is positive definite $\left({ }^{20}\right)$.

THEOREM 4.2. If the symmetric and positive definite system (4.9) satisfies the assumptions of Theorem 4.1 then it admits at least one solution.

Proof. This theorem is an immediate consequence of Theorem 4.1 since in the symmetric and positive definite case $\left.{ }^{21}\right)$ we may assume $\phi_{\rho}(s)=\phi_{\rho}{ }^{*}(s)$, $y(s)=y^{*}(s), y_{j}(s)=y_{j}^{*}(s)$.

5. Concluding remarks. As stated in the introduction, Theorem 4.2 was first proved by Golomb under somewhat stronger assumptions $\left({ }^{22}\right)$. If special-

(19) Obviously $\Phi_{1}^{*}(h)=\phi_{\rho}^{*}(s)$ if $h$ is the element of $H_{1}$ whose components are all zero except for the $\rho$ th one which is 1 .

(20) As is verified immediately from (3.1) this is equivalent to saying that (4.9) is symmetric if $K_{i j}(t, s)=K_{i i}(s, t)$, and positive definite if for all continuous $y_{j}(s)$, $\sum_{i=1}^{n} \sum_{j=1}^{n} \int_{D_{0}} \int_{D_{0}} K_{i j}(s, t) y_{i}(s) y_{j}(t) d s d t \geqq 0$. Cf. [4, p. 54, 55].

(21) Symmetry alone is not sufficient for $\phi_{\rho}(s)=\phi_{\rho}^{*}(s)$.

(22) His Theorem 1 in [4] requires in addition to the assumptions of the present paper continuity of the kernels $K_{i j}(s, t)$ and differentiability of the $f_{i}$ 's. Golomb notices that these conditions are not sufficient to assure uniqueness; by adding a new condition he obtains also uniqueness results. 
ized to the case of one single equation the assumptions of Theorem 4.2 are exactly those of Hammerstein [6, Theorems 1 and 2] with the following exception: the assumption (3.7) of the present paper takes the place of Hammerstein's assumption [6, Theorem 1] of an admissible and bounded kernel. Therefore to show that Theorem 4.2 actually contains the Hammerstein results we have to prove the following lemma.

Lемма 5.1. Let the symmetric and positive definite kernel $K(s, t)$ be admissible and bounded in the admissible domain D. Then the assumption (A) (that is, (3.7)) is satisfied $\left({ }^{23}\right)$.

Proof. Let

$$
|K(s, t)| \leqq C^{2}
$$

and set

$$
K_{n}(s, t)=\sum_{i=1}^{n} \lambda_{i} \phi_{i}(s) \phi_{i}(t)
$$

where as always the $\phi_{i}(s)$ are the eigenfunctions of $K(s, t)$. Then $K(s, t)$ $-K_{n}(s, t)$ is positive definite (cf. $[2$, chap. $3, \S 4.2]$ ) and therefore by $(5.1)$

$$
\begin{aligned}
\int_{D} \int_{D} K_{n}(s, t) y(s) y(t) d s d t & \leqq \int_{D} \int_{D} K(s, t) y(s) y(t) d s d t \\
& \leqq C^{2}\left(\int_{D} y(s) d s\right)^{2}
\end{aligned}
$$

for all non-negative $y(s)$. To prove (3.7) it will be sufficient to show that

$$
K_{n}(s, s) \leqq C^{2}
$$

for all $n$. If this inequality were wrong we would have $K_{n}\left(s_{0}, s_{0}\right)>C^{2}$ for some $s_{0}, n$. Let then $\bar{C}$ be such that for such $s_{0}, n$

$$
K_{n}\left(s_{0}, s_{0}\right)>\bar{C}^{2}>C^{2} .
$$

Since $K_{n}(s, t)$ is continuous we shall have $K_{n}(s, t) \geqq \bar{C}^{2}$ for all points $s, t$ of $D$ in a small enough spherical neighborhood $U$ of $s_{0}$. Let now $y(s)$ be a continuous function which is zero outside $U$, non-negative in $U$ and not identically zero. Then by (5.2)

$$
\bar{C}^{2}\left[\int_{D} y(s) d s\right]^{2} \leqq \int_{D} \int_{D} K_{n}(s, t) y(s) y(t) d s d t \leqq C^{2}\left[\int_{D} y(t) d t\right]^{2}
$$

which implies $\bar{C}^{2} \leqq C^{2}$ in contradiction to (5.3).

${ }^{(23)}$ In the case of a continuous kernel this would be an immediate consequence of Mercer's theorem. 


\section{BIBLIOGRAPHY}

1. L. Alaoglu, Weak topologies of normed linear spaces, Ann. of Math. vol. 41 (1940) pp. 252-267.

2. R. Courant and D. Hilbert, Methoden der mathematischen Physik, vol. 1, 2d ed., Berlin, Springer, 1931.

3. M. Golomb, Zur Theorie der nichtlinearen Integralgleichungen, Integralgleichungssysteme und allgemeinen Funktionalgleichungen. Math. Zeit. vol. 39 (1934) pp. 45-75.

4. - Ueber Systeme von nichtlinearen Integralgleichungen, Publications Mathématiques de l'Université de Belgrade, vol. 5, 1936, pp. 52-83.

5. A. Hammerstein, Nichtlineare Integralgleichungen und direkte Methoden der Variationsrechnung, Sitzungsberichte der Berliner Mathematischen Gesellschaft vol. 26 (1927) pp. 66-70.

6. - Nichtlineare Integralgleichungen nebst Anwendungen, Acta Math. vol. 54 (1930) pp. 118-176.

7. E. H. Rothe, Gradient mappings in Hilbert space, Ann. of Math. vol. 47 (1946) pp. 580592.

8. - Completely continuous scalars and variational methods, Ann. of Math. vol. 49 (1948) pp. 265-278.

9. — Gradient mappings and extrema in Banach spaces, Duke Math. J. vol. 15 (1948) pp. $421-431$.

10. E. Schmidt, Zur Theorie der linearen und nichtlinearen Integralgleichungen, I. Teil, Math. Ann. vol. 63 (1907) pp. 433-476.

11. - Zur Theorie der linearen und nichtlinearen Integralgleichungen, III. Teil, Math. Ann. vol. 65 (1908) pp. 370-399.

UNiversity OF Michigan, AnN Arbor, Mich. 